\title{
RNAi suppressor: The hidden weapon of SARS-CoV
}

\author{
Sumona KarJeE ${ }^{1} *$ (i) and Sunil Kumar MukherJeE ${ }^{2}$ \\ ${ }^{1}$ Molecular Biology Department, Prantae Solutions Private Limited, Bhubaneswar 751 024, India \\ ${ }^{2}$ Division of Plant Pathology, Indian Agricultural Research Institute, New Delhi 110 012, India \\ *Corresponding author (Email, sumonakarjee@gmail.com)
}

MS received 16 May 2020; accepted 6 July 2020; published online 16 July 2020

\begin{abstract}
The two biological evidences to endorse the antiviral activity of RNA interference (RNAi) are biogenesis of viral-siRNA (v-siRNA) by the host and encoding of RNAi-suppressor protein by viral genome. It has been recently established that mammals and mammalian cell lines mount antiviral RNAi to defend themselves against the invading viruses. The large part of viral pathogenicity is also due to the RNAi suppressor proteins. In this context it is only natural to ask what kinds of RNAi suppressors are encoded by the Severe Acute Respiratory Syndrome Coronavirus 2 (SARS-CoV-2), the central character of the present pandemic. The following mini review addresses this question.
\end{abstract}

Keywords. Coronavirus; counter-defense; RNA interference; RNAi suppressor; SARS-CoV; v-siRNA

Host-pathogen interaction in any pathogenesis is a tug of war, where the disease outcome depends upon how effectively the pathogen counters the host defense mechanism. Viruses are Nature's smartest of organisms that have evolved astonishing counter-defense strategies. This is quite evident from the fact that within a matter of a few months, the SARS-CoV-2 has held the entire world hostage with more than 10 million infections and 5.2 lakh casualties. Each passing day, we are getting new insights about its etiology, but here we will focus on its smart counter-defense capability of encoding RNA interference (RNAi) suppressor. The immune system of an organism plays a vital role in protection both through innate and adaptive immunity. The diversity of non-self-recognition molecules in innate immunity is germline encoded and provides non-specific protection against pathogens, whereas the adaptive immunity is a product of somatic diversification and selective clonal expression and is entirely pathogen specific. The innate immune defense

This article is part of the Topical Collection: COVID-19: Disease Biology \& Intervention. includes: (1) physical obstructions like epithelial barriers; (2) production of antimicrobial serum proteins such as complement; (3) natural antibodies produced by B1 lymphocytes; (4) the phagocytic activities of cells such as neutrophils, macrophages, and dendritic cells; (5) natural killer (NK) cells that can lyse virusinfected cells; (6) various cell types present at sites of virus invasion, possessing receptors to recognize and quickly respond to invading viruses employing transcriptional activation leading to a wide variety of protective molecules, and the interferon (IFN) system plays the lead role of such antiviral resistance; (7) apoptosis, a process of programmed cell death that can eliminate virus-infected cells (MacLachlan and Dubovi 2011). The adaptive immunity, on the other hand, can be subdivided further into two major types depending on mode of induction of immune response: the humoral one based on immunoglobulin (Ig) secreted by B lymphocytes, and the other one being T-cell-based immune system, which generates the histocompatibility complex (MHC)-restricted antigen that are presented to the $\mathrm{T}$ cell receptor (TCR) on T lymphocytes (Yuan et al. 2014). Antiviral immunity is also provided by many antiviral cellular proteins like ZAP, which binds 
specifically at the $\mathrm{CpG}$ dinucleotide and recruits other proteins to degrade the viral RNA genome (Xia 2020). It is interesting to note that many RNA viruses including SARS-CoV-2 have evolved to have $\mathrm{CpG}$ deficiency in order to avoid action of such antiviral proteins. In addition, there is a panorama of peptides derived from animal venom that can serve as antiviral agents (da Mata et al. 2017). Beside these, eukaryotic cells have evolved another interesting line of defense against viral attack, namely RNAi mechanism which helps generate silencing RNAs called small interfering RNA (siRNAs) from all over the viral genome (vsiRNAs) that in turn incapacitate the viral transcripts. This mechanism is very vital, especially considering that the immune system can be breached by smart viruses pretty easily that are infecting the host for the first time. Ironically, viruses have also co-evolved to encode special suppressor activity in their proteins with an aim to foil the efforts of RNAi-mediated host defense. The disease outcome, thus, is the balance between these two opposing forces, namely the host RNAi factors and the counter defending viral RNAisuppressors. This general dogma has been validated in almost all plant viruses. But the story is different for animal viruses. For long, it was held that the animal viruses are not subject directly to RNAi-mediated destruction. This notion persisted even though that many viruses, including HIV, Ebola, etc., were found to encode RNAi-suppressors. We at ICGEB, New Delhi, showed that RNAi-suppressors are encoded by SARS7a (Karjee et al. 2010), Dengue-NS4B (Kakumani et al. 2013), HBV-X (Chinnappan et al. 2014; Ghosh et al. 2017) ORFs. The missing component of the puzzle was the demonstration of v-siRNAs from the animal viral genome. Parameswaran et al. (2010) first demonstrated generation of $\mathrm{v}$-siRNAs in suitable cell lines. However, in initial experimental evidences the titre of v-siRNAs was very low and these v-siRNAs were uncharacterized. The convincing breakthrough appeared in reports that occurred between years of 2013 and 2017 (Jailani and Mukherjee 2017; Maillard et al. 2019). In one such study, Li et al. (2013) detected v-siRNAs in mammalian cells and young mice which were challenged with the Nodamura virus. This virus encodes a strong RNAi-suppressor called B2, and high accumulation of $\mathrm{v}$-siRNAs occurred in the absence of the B2 suppressor. Similar studies were reported later in many animal viruses (Jailani and Mukherjee 2017; Maillard et al. 2019). These v-siRNAs were dicer dependent, loaded correctly in AGO2 complexes to slice the homologous viral transcripts. The difficulties in detecting animal viral v-siRNAs were compounded by the interferon systems present in animal cells (Maillard et al. 2019). Thus, there is a uniformity of antiviral RNAi activities across the kingdoms. However, system-specific uniqueness is always there. In all of these cases of disease induction, contributions of microRNAs have been left out of the equations for the sake of simplicity. How do the RNAi-suppressors help the viruses? The mutant viruses lacking the suppressors are toothless in terms of their survival and disease inductions (Maillard et al. 2019, Sanan-Mishra et al. 2017). In case of plants, the suppressors alone can cause disease symptoms in the absence of virus when expressed ectopically in plant tissues. Hence, the suppressor proteins encoded by the viral genome are regarded as the pathogenic factors (Sanan-Mishra et al. 2017). The viral suppressors do not have any recognizable general motif in them and act at various steps of RNAi-activities, biochemically (Sanan-Mishra et al. 2017). In the last few months, pandemics of COVID19 have been observed all over the globe in a very devastating manner. Thus, the obvious question that comes up whether the SARS-CoV-2 codes for any RNAi-suppressor.

The SARS-CoV virus has been reported to encode for RNAi suppressor - in fact, not one but two different suppressors till date. The first reported RNAi suppressor from SARS-CoV was reported by our group in 2010, when we identified SARS-7a protein as the suppressor protein (Karjee et al. 2010). As RNAi suppressor are known to act across kingdoms, the initial demonstration of suppression activity was carried out in a model plant system with the GFP reporterbased reversal of silencing assay and replication-based spot assay (figure 1A and B). The strength of SARS-7a suppression activity was comparable to established strong RNAi suppressor of plant viral origin, namely Mungbean yellow mosaic India virus (MYMIV)-AC2 and an insect viral origin Flock house virus (FHV)-B2. The suppression activity was further validated in mammalian cell system with reversal of silencing assay (figure 1A) as well as replication enhancement assay of Tat-mutated (RNAi suppressor of HIV) HIV-1 replicon in HEK293T and SARS-CoV-permissive human lung epithelial cell line A549 (figure 1B). This suggests that SARS-CoV-7a protein is a strong RNAi suppressor, implicating its role in the pathogenicity of the virus.

The amino acid sequence of SARS-7a protein does not exhibit any significant similarity to any other viral or non-viral proteins but has been conserved in all SARS-CoV species (Li et al. 2005). The sequence homology of $7 \mathrm{a}$ protein of SARS-CoV and SARS- 


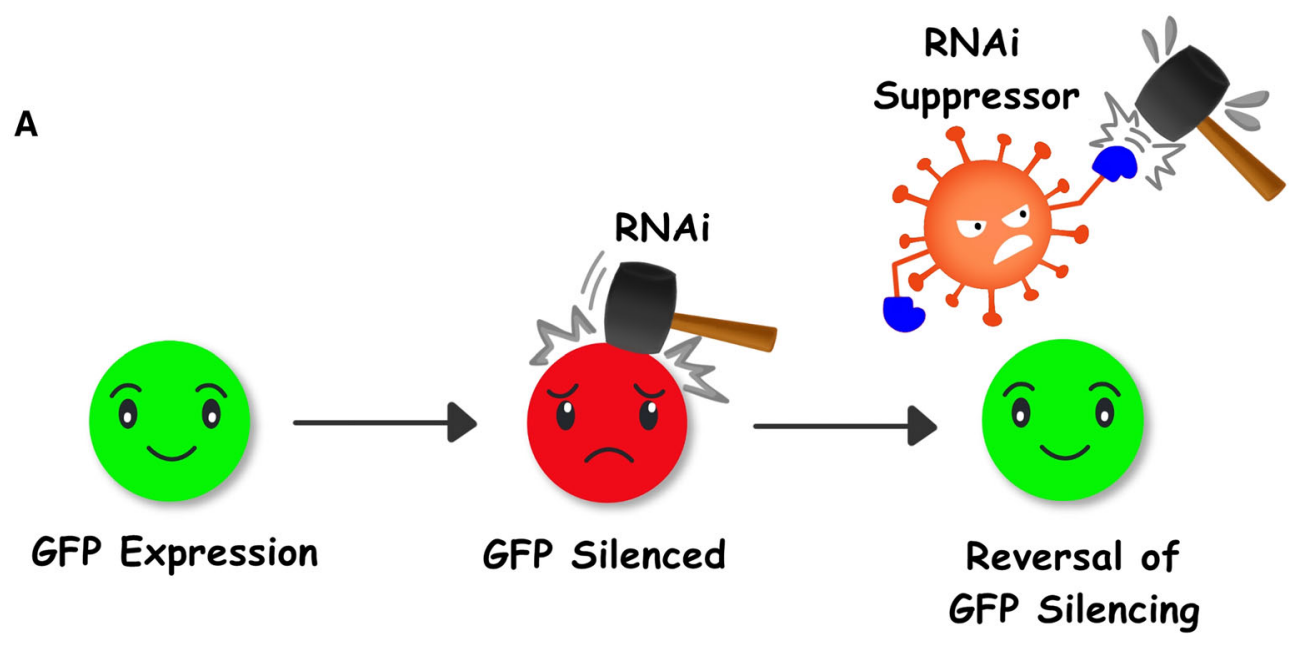

B

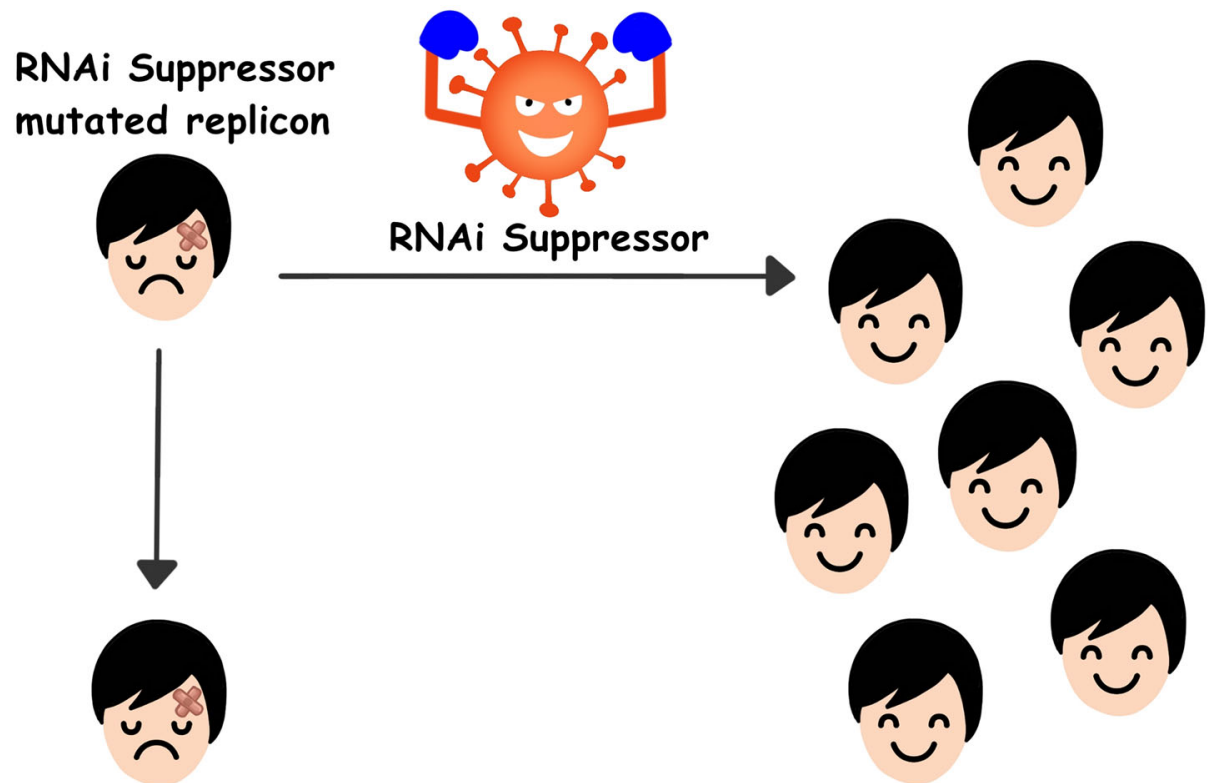

\section{Low number of replicons}

High number of replicons

Figure 1. Cartoon representation of RNAi suppression activity through (A) Reversal of Silencing assay and (B) Replication based spot assay.

CoV-2 is also found to be very high, i.e. about $90 \%$, as determined by NCBI blast result. Hence, we suggest that the SARS-CoV-2-7a protein might have RNAi suppression activity as well, although it needs to be validated experimentally.

The sequence analysis of ORF $7 \mathrm{a}$ indicates it as a type I transmembrane protein composed of 122 amino acids residues with 15-residue $\mathrm{N}$-terminal signal peptide, 81-residue-long luminal domain, a 21-residuelong transmembrane segment, and a 5-residue cytoplasmic tail. The experimental domain analysis for the RNAi suppression activity of the SARS-CoV-7a protein showed that the central domain with the smallest fragment studied from aa $32 \rightarrow 89$ exhibited RNAi suppression activity (Karjee et al. 2010) and this domain is also highly conserved in SARS-CoV-2. Interestingly, this fragment overlaps the 'luminal' domain of the $7 \mathrm{a}$ that shows topology similarity with the members of the immunoglobulin superfamily as revealed in the resolved crystal structure of the $7 \mathrm{a}$ protein (Nelson et al. 2005). The localization studies on 7 a reveal that it is present intracellularly, primarily with endoplasmic reticulum and Golgi compartments. It is noteworthy that the positive-stranded SARS-CoV genome replicates through dsRNA intermediate that has also been found to be localized in ER and Golgi 


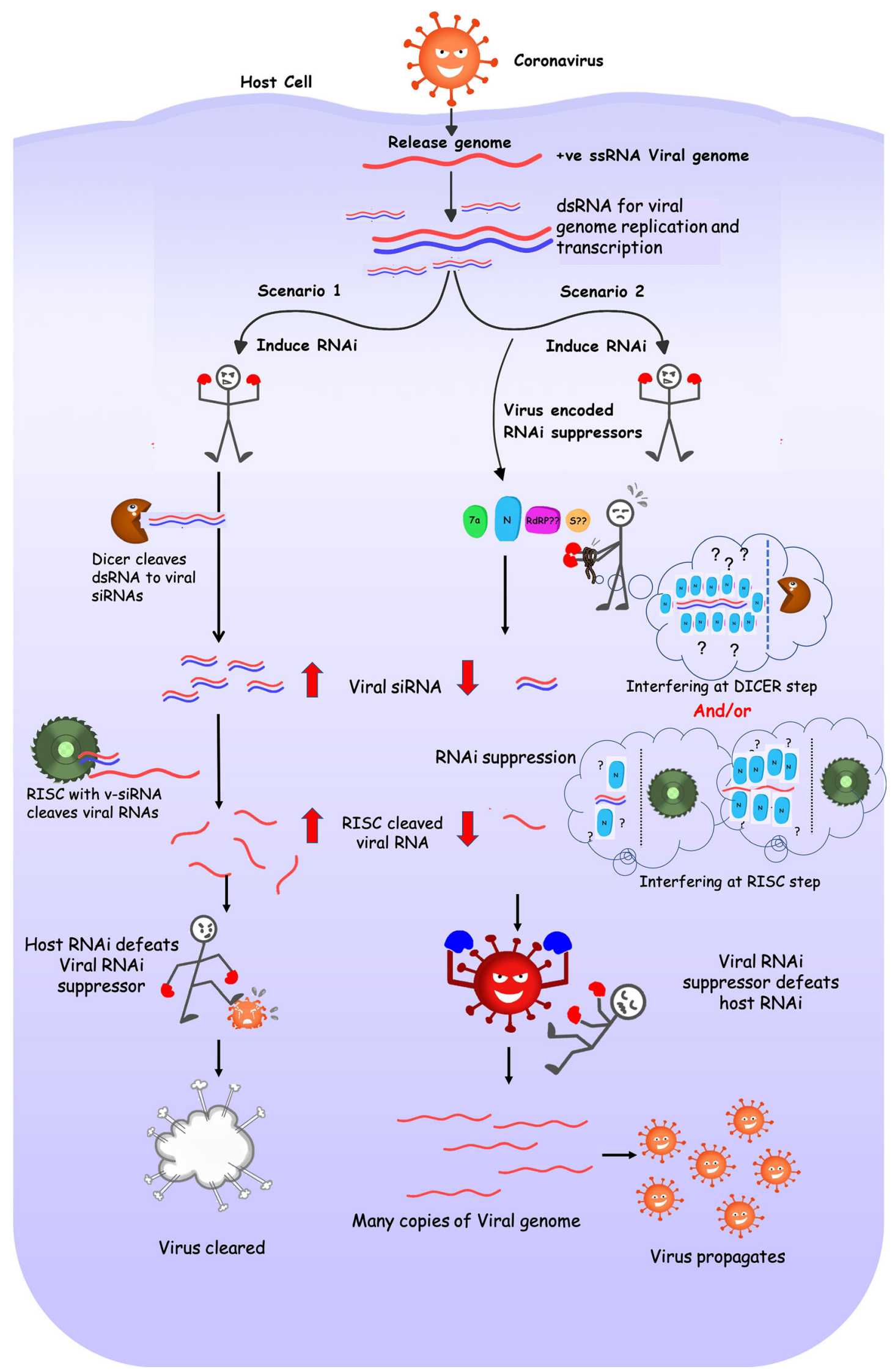


4Figure 2. Schematic representation of disease outcome with host defense mechanism RNAi and viral counter defense RNAi suppressors.

complex (Knoops et al. 2008). The unprotected and uncomplexed dsRNA has been the major trigger for the induction of RNAi to generate viral specific siRNAs. Interestingly, the rough endoplasmic reticulum is also known to be central nucleation site of siRNA-mediated RNA silencing (Stalder et al. 2013). Thus, the localization of 7a in ER and Golgi complex might be coupled to its function of RNAi suppression activity. However, the detailed mechanism for its RNAi suppression is yet to be decoded.

Many of the highly pathogenic viruses encode for more than one RNAi suppressor and probably SARS is no exception. The second SARS-encoded RNAi suppressor that has been experimentally validated is nucleocapsid $(\mathrm{N})$ protein. The RNAi suppression activity of N-protein has been first reported in 2015 by Guo and Chen group from Wuhan University, Wuhan, Hubei, China (Cui et al. 2015). The RNAi suppression activity of N-protein was determined using the reversal-of-silencing assay in HEK293T cells (figure 1A). The RNAi suppression activity has been demonstrated to be not limited to protein level but has also been exhibited by corresponding N-mRNA. Similar to $7 \mathrm{a}$ studies, they have also conducted replication enhancement assay (figure 1B) in insect cell line S1 with B2-deficient mutant (pRNA1- $\Delta \mathrm{B} 2$ ) replication system, where the co-transfection of $\mathrm{N}$-encoding plasmid resulted in partial rescue of the FHV RNA1 and RNA3, which was otherwise insignificantly detectable. The N-protein due to its RNA binding ability exhibits suppression activity both at Dicer-processing and postDicer stages, as the positively charged residues Lys 258 and Lys 262 of SARS-CoV N-protein are critical for the RNAi repression activity. The $\mathrm{C}$-terminal domain of N-protein of SARS-CoV-2 has the basic region between residues 248 to 280 that forms a positively charged groove with likely binding region for RNA (Chen et al. 2007). Recently, in a letter to editor of Science China Life Sciences from Wuhan University, Zhou's group claimed the RNAi suppressor activity of $\mathrm{N}$-protein of the pandemic strain of SARS-CoV-2 $(\mathrm{Mu}$ et al. 2020), employing reversal of silencing assay. To evaluate the strength of SARS CoV N-protein as RNAi suppression activity vis-à-vis host RNAi defense, it will be interesting to compare the binding affinity towards viral RNA (the ds form) between viral
$\mathrm{N}$-protein and host Dicer complexed with its cognate RNA binding protein TRBP.

The RNA genome of SARS-CoV-2 is about $30 \mathrm{~K} \mathrm{nt}$ long and a genome of that large size might have the potential to encode multiple suppressors to reinforce its pathogenic character (figure 2). Although the RNAisuppressor proteins do not have any general motif, a subset of these is characterized by repeats of $\mathrm{GW}$ (Glycine-Tryptophan) or WG motifs in their amino acid sequences. The proteins having such repeats often interact with $\mathrm{AGO} 2$ proteins and transport them in P-bodies, thus blocking the RISC functions. We searched for these motifs in the various ORFs of SARSCoV-2 viral genome. Two ORFs stood out as the candidate proteins. The full RdRP of the virus or the lab ORF harbors three GW and two WG motifs. Similarly, the spike protein has also three GW repeats. Thus, these ORFs might serve as potential RNAi-suppressors; however, this conjecture needs to be experimentally validated.

Can we downregulate the suppressors and force the virus to lose its steam? Judicious approaches by RNAi and CRISPR-Cas13a might give us the desired goal. Moreover, the virus upon entering the mammalian cells might change the endogenous siRNA profile of the host. The changed siRNAs are known as virus-activated siRNAs or va-siRNAs. Some of these siRNAs will be antiviral but some might still work as proviral. These proviral siRNAs and their host targets need to be identified so that these proviral va-siRNAs could be downregulated or their targets could be upregulated to make the cells tolerant of the infecting corona viruses.

\section{Acknowledgements}

The authors acknowledge Dr. Aseem Mishra for his contribution in editing and Ms. Susrita Samantray for her contribution in the illustration creation.

\section{References}

Chen CY, Chang CK, Chang YW, Sue SC, Bai HI, Riang L, Hsiao CD and Huang TH 2007 Structure of the SARS coronavirus nucleocapsid protein RNA-binding dimerization domain suggests a mechanism for helical packaging of viral RNA. J. Mol. Biol. 368 1075-1086

Chinnappan M, Singh AK, Kakumani PK, Kumar G, Rooge SB, Kumari A, Varshney A, Rastogi A, Singh AK, Sarin SK and Malhotra P 2014 Key elements of the RNAi pathway are regulated by hepatitis $\mathrm{B}$ virus replication and 
HBx acts as a viral suppressor of RNA silencing. Biochem. J. 462 347-358

Cui L, Wang H, Ji Y, Yang J, Xu S, Huang X, Wang, Z, Qin L, Tien P, Zhou X and Guo D 2015 The nucleocapsid protein of coronaviruses acts as a viral suppressor of RNA silencing in mammalian cells. J. Virol. 89 9029-9043

da Mata EC, Mourao CB, Rangel M and Schwartz EF 2017 Antiviral activity of animal venom peptides and related compounds. J. Venom. Animal. Toxins Trop. Dis. 233

Ghosh S, Kaushik A, Khurana S, Varshney A, Singh AK, Dahiya P, Thakur JK, Sarin SK, Gupta D, Malhotra P and Mukherjee SK 2017 An RNAi-based high-throughput screening assay to identify small molecule inhibitors of hepatitis B virus replication. J. Biol. Chem. 292 12577-12588

Jailani AAK and Mukherjee SK 2017 Does endogenous RNAi have antipathogenic features? Ind. J. Plant Physiol. 22 506-513

Kakumani PK, Ponia SS, Sood V, Chinnappan M, Banerjea AC, Medigeshi GR, Malhotra P, Mukherjee SK and Bhatnagar RK 2013 Role of RNA interference (RNAi) in dengue virus replication and identification of NS4B as an RNAi suppressor. J. Virol. 87 8870-8883

Karjee S, Minhas A, Sood V, Ponia SS, Banerjea AC, Chow VT, Mukherjee SK and Lal SK 2010 The 7a accessory protein of severe acute respiratory syndrome coronavirus acts as an RNA silencing suppressor. J. Virol. 84 10395-10401

Knoops K, Kikkert M, van den Worm SH, ZevenhovenDobbe JC, van der Meer Y, Koster AJ, Mommaas AM and Snijder EJ 2008 SARS-coronavirus replication is supported by a reticulovesicular network of modified endoplasmic reticulum. PLoS Biol. 6 e226

Li W, Shi Z, Yu M, Ren W, Smith C, Epstein JH, Wang H, Crameri G, Hu Z, Zhang H and Zhang J 2005 Bats are natural reservoirs of SARS-like coronaviruses. Science $310676-679$

Corresponding editor: ManchiKatla Venkat Rajam
Li, Y, Lu J, Han Y, Fan X and Ding SW 2013 RNA interference functions as an antiviral immunity mechanism in mammals. Science 342 231-234

MacLachlan NJ and Dubovi EJ 2011 Fenner's veterinary virology (London Academic Press) pp 75-99

Maillard PV, Van der Veen AG, Poirier EZ and Sousa CR 2019 Slicing and dicing viruses: antiviral RNA interference in mammals. EMBO J. 38 e100941

Mu J, Xu J, Zhang L, Shu T, Wu D, Huang M, Ren Y, Li X, Geng Q, Xu Y and Qiu Y 2020 SARS-CoV-2-encoded nucleocapsid protein acts as a viral suppressor of RNA interference in cells. Sci. China Life Sci. https://doi.org/ 10.1007/s11427-020-1692-1

Nelson CA, Pekosz A, Lee CA, Diamond MS and Fremont DH 2005 Structure and intracellular targeting of the SARS-coronavirus Orf7a accessory protein. Structure $\mathbf{1 3}$ $75-85$

Parameswaran P, Sklan E, Wilkins C, Burgon T, Samuel MA, Lu R, Ansel KM, Heissmeyer V, Einav S, Jackson W and Doukas T 2010 Six RNA viruses and forty-one hosts: viral small RNAs and modulation of small RNA repertoires in vertebrate and invertebrate systems. PLoS Pathogens 6 e1000764

Sanan-Mishra N, Chakraborty S, Gupta D and Mukherjee SK 2017 RNAi suppressors: biology and mechanisms; in Plant epigenetics (Springer, Cham) pp 199-230

Stalder L, Heusermann W, Sokol L, Trojer D, Wirz J, Hean J, Fritzsche A, Aeschimann F, Pfanzagl V, Basselet P and Weiler J 2013 The rough endoplasmatic reticulum is a central nucleation site of siRNA-mediated RNA silencing. EMBO J. 32 1115-1127

Xia X 2020 Extreme genomic CpG deficiency in SARSCoV-2 and evasion of host antiviral defense. Mol. Boil. Evol. https://doi.org/10.1093/molbev/msaa094

Yuan S, Tao X, Huang S, Chen S and Xu A 2014 Comparative immune systems in animals. Annu. Rev. Animal Biosci. 2 235-258 